\title{
Anti-predation Defense Traits of Daphnia are Associated With the Gut Microbiota Composition Shaped by Fish Kairomone
}

Qi Liu

Nanjing Normal University

Siddiq Akbar

Nanjing Normal University

Zihao Ding

Nanjing Normal University

Qiming Zhou

Nanjing Normal University

Lei Gu

Nanjing Normal University

Yunfei Sun

Nanjing Normal University

Zhou Yang ( $\nabla$ yangzhou@njnu.edu.cn )

Nanjing Normal University https://orcid.org/0000-0001-9664-7105

Research

Keywords: Daphnia magna, Gut microbiota, Host-microbiota interaction, Predation risk, Inducible defense, Life-history traits

Posted Date: August 3rd, 2021

DOI: https://doi.org/10.21203/rs.3.rs-757585/v1

License: (c) (1) This work is licensed under a Creative Commons Attribution 4.0 International License. Read Full License 
1 Anti-predation defense traits of Daphnia are associated with the gut microbiota

2 composition shaped by fish kairomone

3 Qi Liu, Siddiq Akbar, Zihao Ding, Qiming Zhou, Lei Gu, Yunfei Sun, Zhou Yang*

4 Jiangsu Key Laboratory for Biodiversity and Biotechnology, School of Biological

5 Sciences, Nanjing Normal University, 1 Wenyuan Road, Nanjing 210023, China

6

$7 \quad$ *Corresponding author: Zhou Yang

8 Jiangsu Key Laboratory for Biodiversity and Biotechnology, School of Biological

9 Sciences, Nanjing Normal University, 1 Wenyuan Road, Nanjing 210023, China

10

11 E-mail:yangzhou@njnu.edu.cn

12 


\section{Abstract}

Background: Gut microbiota plays an important role in host physiology and fitness.

The gut microbiota can promote host health by influencing life history traits, especially in arthropods. However, it is not clear whether the performance of host defense traits in response to predator pressure in natural food webs is related to their gut microbiota composition. In this study, we used Daphnia magna as a model organism to investigate the relationship of $D$. magna life history traits and gut microbiota alterations under predator kairomone based on $16 \mathrm{~S}$ rRNA amplicon sequencing.

Result: We showed that the microbiota composition of $D$. magna was significantly affected by their predator risk and development stage. The relative abundance of Comamonadaceae (mainly Limnohabitans sp.) significantly decreased in the presence of predator kairomone. Furthermore, the presence of predator kairomone significantly reduced the $\alpha$ diversity of gut microbiota in D. magna with the increase of instar. Among them, the OTUs belonged to Epsilonbacteraeota and Firmicutes in the presence of predator kairomone were significantly higher than those in the control group. The results of functional predictions showed that predation pressure promote the metabolic function of gut microbiota, such as metabolism of energy, cofactors, and vitamins. By analyzing the correlation between the induced defense traits of $D$. magna and the relative abundance of bacteria, we found that the increased abundance of Comamonadaceae, Moraxellaceae, and Flavobacteriaceae were linearly correlated with the partial defense traits of D. magna. Specifically speaking, body size was 
positively correlated with an increased abundance of Comamonadaceae, whereas spine length was negatively correlated with an increased abundance of Comamonadaceae but was positively correlated with increased Flavobacteriaceae abundance.

Conclusions: Our results suggested that predation risk can affect the composition of the gut microbiota in D. magna, which may indirectly induce the production of defensive traits in D. magna. The results of this study revealed an important role of gut microbiota in the development of defensive traits of Daphnia in response to fish predators. The correlation between microbial abundance and defense traits is of great significance for further understanding the effect of host-microbiota interaction on individual anti-predation defense.

Keywords: Daphnia magna, Gut microbiota, Host-microbiota interaction, Predation risk, Inducible defense, Life-history traits 


\section{Background}

The digestive tract of animals is host to a diverse community of symbiotic microorganisms, collectively called the gut microbiota. Evidence has accumulated that the gut microbiota is not just a random set of microorganisms, but rather a complex community that plays a critical role in host physiology and behavior [1-3]. Some studies have shown that gut microbiota may also contribute to host health by influencing life history traits of the host, in particular in arthropods, which reveal that gut bacteria have an over-whelming influence on growth, development, reproduction and survival. In the water flea Daphnia magna $[4,5]$ and in the fruit fly Drosophila melanogaster [6], germ-free individuals develop more slowly and are smaller than conventional animals, while in mosquitoes, axenic larvae fail to develop beyond the first instar [7]. In all these species, inoculating axenic larvae with gut bacteria can restore a normal developmental rate [5-7]. As a result, the gut microbiota is increasingly seen as a key driver of health.

Microbiome is highly plastic, and can respond rapidly to changes in host diet or environmental conditions, through changes in community composition, mutations, exchange of genetic material with bacteria from the environment, or changes in gene expression [8-10]. In natural animal populations, there is a relationship between gut microbial composition and their host nutritional status in the food web [11]. One important aspect of nutritional status affecting gut microbiota is related to shifts in diet quality and quantity [5, 12]. Akbar et al. [13] discovered that gut microbiota composition of D. magna is significantly different under different food conditions, 
and the abundance of Pseudomonadaceae in gut microbiota of D. magna decreases significantly under lower food quality. Callens et al. [5] found that when food is sufficient or abundant, the microbiota has a strong positive effect on the growth and reproduction of cladocerans, while in the case of limited food, the microbiota has a weak effect on the growth and reproduction of Daphnia. In nature, however, in addition to changes in food quality and quantity, predation is also an important factor in the food chain. Increasing or decreasing predator density will change prey densities and indirectly affect the food resources availability for the prey [14-16]. On the other hand, chemical cues from predators have been shown to reduce activities in prey and subsequently induce prey morphological changes that could decrease the risk of being predated [17-19]. Moreover, predation stress also influences the physiological status of prey, for example, hormones released from stress could mediate immunological and behavioral responses in vertebrates [20]. It has been shown that nerve and immune system can play important roles in regulating gut microbiota communities $[21,22]$. Gut microbiota provide their host with metabolic capabilities not directly encoded in the host genome, such as digestion of plant polysaccharides [23] or detoxification of food borne toxins $[24,25]$, and contribute to the normal development of the host, e.g. by fostering the maturation of the immune system [26]. Thus, changes the composition of host gut microbiota may not only depend on shifts in nutritional status, but also on the risk of predation.

In addition, gut microbiota is also affected by developmental stages [27, 28]. For example, Moll et al. [29] showed that newly emerged mosquito adults contain few or 
no bacteria in their guts. Shifts in the microbial community in Bombyx mori are apparent between early- and late-instar larvae, in concert with host developmental changes [30]. The diversity and composition of amphibian gut microbial communities differ between tadpoles and adults [31]. Further, studies have shown that the gut microbiota communities are initially assembled by neutral or stochastic processes, and become more fixed and stable later in host development, indicating an increased importance of selective forces shaping the gut microbiome as animal development proceeds [32-34]. Microbes will be selectively recruited through interactions with the host and the already established microbiota [35], and then gradually create a stable microbiome that is adapted to the environment.

Predators play an important ecological role in ecosystem, and the relationship between predators and prey has been extensively studied [36, 37]. However, under predation risk, does the gut microbiota of prey change with developmental stage during the defense process? No study has reported on this issues. The freshwater crustacean D. magna provide opportunity for such studies. D. magna has high experimental traceability, short life cycle, strong clone reproduction, and rapid response ability to environmental stress. Previous studies have shown that D. magna can respond to chemicals released by their predators and exhibit different types of defense, e.g., alterations in morphologies [38, 39], adjustment in life-history strategy [40]. In this study, we used D. magna as the model organism and investigated the effects of the presence of fish predation risk on the gut microbiota composition of $D$. magna at different instars. We hypothesized that: (1) Kairomone released by fish 
predators alters the relative abundance of the D. magna gut microbiota; (2) The development stage of D. magna can interact with fish kairomone to affect the diversity of the gut microbiota community; (3) Changes in D. magna defense traits are related to the composition of D. magna gut microbiota community under the influence of fish kairomone. To test these hypotheses, we placed D. magna in the medium with and without fish kairomone, and recorded key life history traits as well as the composition of the gut microbiota community of D. magna at different instars.

\section{Methods}

\section{Experimental organisms and cultivation}

Laboratory-cultured clone of $D$. magna that have been maintaining in laboratory under standard conditions for more than ten years were used in our experiments. Stock Daphnia clonal lineage was cultured in COMBO medium (refreshed four times a week) at a temperature of $25{ }^{\circ} \mathrm{C}$ under fluorescent light at $50 \mu \mathrm{mol}$ photons $\mathrm{m}^{-2} \mathrm{~s}^{-1}$ with a $14: 10 \mathrm{~h}$ light : dark cycle. They were fed daily with high-quality food green alga Scenedesmus obliquus $\left(1.5 \mathrm{mg} \mathrm{C} \mathrm{L}^{-1}\right)$, which was harvested from that cultured in $1 \mathrm{~L}$ of BG-11 medium in Erlenmeyer flasks under the same conditions.

\section{Kairomone production}

For harvesting fish kairomones, 14 small-sized fish (Rhodeus ocellatus, $3-5 \mathrm{~cm}$ in body length) were cultured in COMBO medium and fed with about $5000 \mathrm{D}$. magna. After $6 \mathrm{~h}$, the fishes were transferred into $7 \mathrm{~L}$ medium to excrete kairomone for $18 \mathrm{~h}$ without any other food supply [41]. In order to prevent bacterial degradation, 
the kairomone medium was filtered through a $0.22 \mu \mathrm{m}$ glass fiber filter (Millipore, USA), and then stored at $-20{ }^{\circ} \mathrm{C}$ until further usage.

\section{Experimental design}

Before the experiment, reproduction of the animals was synchronized by maintaining individuals of the same age for at least two generations under identical conditions. We used the third brood neonates born within $24 \mathrm{~h}$ for experiments to ensure that the test animals would be treated for the majority of their juvenile period, exclude the maternal effects [42], and minimize differences between experimental animals [43].

We set up the experiments with absence (control) and presence (treatment) of fish kairomone. The treatment with fish kairomone were produced by diluting the stock fish kairomone 20 times (i.e., on average one fish per $10 \mathrm{~L}$ ) in the COMBO medium. Each treatment or control contained 250 replicates, and each replicate containing one individual was cultured in $50 \mathrm{~mL}$ of the COMBO medium with the green alga $S$. obliquus as food $\left(1.5 \mathrm{mg} \mathrm{C} \mathrm{L}^{-1}\right)$. The experiment was run for 21 days, and the instar of D.magna was recorded for 21 days. The neonates released by different ages of D.magna were removed after counted, and only the mothers were retained for later microbial analyze.

Some of the first instar D. magna (the newborn ones) and the fourth instar $D$. magna were respectively divided into three samples (each with 15 individuals) for the analysis of microbiota in the whole Daphnia, while some of the sixth, ninth and twelfth instar D. magna were respectively divided into three samples each containing 
5 individuals for this analysis. After removed the Daphnia individuals, the remaining culture medium was filtered by $0.22 \mu \mathrm{m}$ glass fiber filter (Millipore, USA) for the analysis of microbiota in the culture medium [44]. The microbiota was also analyzed in triplicate. Furthermore, at the sixth, ninth and twelfth instar, another 20-25 individuals of D. magna were collected and dissected guts for gut microbial analysis. To isolate the gut, the D. magna were put on sterile glass slide using flame sterilized tweezer, and guts were isolated under stereomicroscope using sterilized tweezer and syringe. Twenty to twenty-five guts were isolated for each sample and were immediately transferred to sterile Eppendorf tubes containing $100 \mu \mathrm{L}$ sterile purified water and kept at $-20{ }^{\circ} \mathrm{C}$ till further analysis. It is worth noting that the gut microbiota was analyzed in duplicate, as it was quite difficult to dissect the gut of D. magna, and thus there was not enough guts for microbiota analysis if we analyzed them in triplicate. In addition, ten D. magna were used for recording the life history traits (i.e., body size, spine length, time to maturation, number of broods, and total offspring number per female) every instar in absence and presence of fish kairomone, and the released neonates were removed after counted.

\section{DNA extraction and PCR amplification}

Microbial DNA was extracted using the HiPure Soil DNA Kits (Magen, Guangzhou, China) according to manufacturer's protocols. The full length 16S rDNA were amplified by PCR $\left(95^{\circ} \mathrm{C}\right.$ for $2 \mathrm{~min}$, followed by 35 cycles of $95^{\circ} \mathrm{C}$ for $30 \mathrm{~s}$, $60{ }^{\circ} \mathrm{C}$ for $45 \mathrm{~s}$, and $72^{\circ} \mathrm{C}$ for $90 \mathrm{~s}$, with a final extension $72^{\circ} \mathrm{C}$ for $10 \mathrm{~min}$ ) using primers 27F: AGAGTTTGATCCTGGCTCAG, 1492R: GNTACCTTGTTACGACTT. 
The PCR reaction was carried out in a $50 \mu \mathrm{L}$ reaction volume with TransGen HighFidelity PCR SuperMix (TransGen Biotech, Beijing, China), $0.2 \mu \mathrm{M}$ forward and reverse primers, and $5 \mathrm{ng}$ template DNA.

Amplicons were evaluted with 2\% agarose gels and purified using the AxyPrep DNA Gel Extraction Kit (Axygen Biosciences, Union City, CA, USA) according to the manufacturer's instructions. Sequencing libraries were generated using SMRTbell TM Template Prep Kit (PacBio, Menlo Park, CA, USA) following manufacturer's recommendation. The library quality was assessed with Qubit 3.0 Fluorometer (ThermoFischer Scientific,USA) and FEMTO Pulse system (Agilent Technologies, Santa Clara, CA, USA). The libraries were sequenced on the PacBio Sequel platform. The raw reads were deposited into the NCBI Sequence Read Archive (SRA) database (Accession Number: SRP******).

\section{Statistical analysis}

The parameters of the response-functions were used to assess the impact of different treatments (presence or absence of fish kairomones) on D. magna body size and spine length / body size change with days. Specifically, we used two predictive functions to fit the data. An exponential rise to an asymptote function, $y=J_{\max }\left(1-\mathrm{e}^{-p t}\right)$, was used to fit the relationships between $D$. magna body size and growth days at presence or absence of fish kairomones, where $y$ is body size of $D$. magna at time $t$, $J_{\max }$ is the predicted asymptotes of the exponential rise functions (maximum value) when $t$ approaches infinity, and $p$ describes the initial rate of change in the response [45]. An exponential decline to an asymptote function, $y=L_{\min }+\alpha \mathrm{e}^{-r t}$, was used to fit 
the relationships between spine length / body size of D. magna and growth days at presence or absence of fish kairomones, where $y$ is spine length / body size of $D$. magna at time $t, L_{\min }$ is the predicted asymptotes of the exponential decline functions (minimum value) when $t$ approaches infinity, and $r$ describes the initial rate of change in the response [46]. The spine length of D. magna showed a "peak curve" trend, i.e., increasing first and then declining, thus a three-parameter Gaussian model: $y=$ $K \times \mathrm{e}^{\left[-0.5\left(\frac{t-t_{k}}{q}\right)^{2}\right]}$ was used to fit the spine length of D. magna, where $y$ represents the spine length of $D$. magna at time $t, K$ represents the theoretical maximum spine length, $t_{k}$ represents the time needed to reach the theoretical maximum spine length, and $q$ reflects the shape of the curve $[47,48]$.

The interactive effect of growth days and presence or absence of fish kairomones on the body size, spine length, and spine length / body size of $D$. magna were assessed using two-way ANOVA $(\alpha=0.05)$. The effect of presence or absence of fish kairomones on time to maturation, body size at maturation, total offspring number, brood number, and average offspring per brood of D. magna were assessed using oneway ANOVA $(\alpha=0.05)$. Post hoc analysis between various treatments and multiple comparisons were determined by Tukey's $\operatorname{HSD}(\alpha=0.05)$. All data were expressed as the mean values $\pm \mathrm{SE}$, and all statistical analyses were performed using Sigmaplot 14.0.

Differences in the abundances of dominant bacterial genera between different growth days with presence or absence of fish kairomones were assessed by analysis of variance (ANOVA) with post-hoc Tukey's test $(\alpha=0.05)$. To observe the overall 
diversity of microbiota, nonmetric multidimensional scaling (NMDS) with BrayCurtis (Vegan 2.3-5) and weighted and unweighted UniFrac distances were calculated using Phyloseq. Spearman's correlation analysis between two variables (bacterial abundance in treatment and life history traits) was performed by bivariate analysis in Sigmaplot 14.0, where a slope that was significantly different from zero $(\alpha=0.05)$ indicated that the bacterial abundance had a significant effect on a given life-history trait. Other bioinformatic analysis was performed using Omicsmart, a real-time interactive online platform for data analysis ( http://www.omicsmart.com).

\section{Results}

\section{Body size, spine length, and reproduction traits}

The time to maturation, body size at maturation, total offspring number, and brood number of D. magna were significantly affected by fish kairomone (Table 1). The body size, spine length, and spine length / body size of $D$. magna were also significantly affected by fish kairomone, and there was significant interaction between kairomone and growth days on these morphological parameters (Table 2). Compared with those not exposed to fish kairomone, the maturation time of D. magna exposed to kairomone was significantly earlier, and the body size at maturity was significantly reduced (Fig. 1, Table 1). Total offspring number and brood number of D. magna exposed to fish kairomone were significantly increased, however, there was no significant difference in the average offspring per brood in D. magna with or without fish kairomone (Fig. 1, Table 1). D. magna exposed to fish kairomone significantly 
decreased body size and increased spine length and spine length / body size compared to those not exposed to fish kairomone (Fig. 2, Table 2). Among them, both the body size and the spine length of $D$. magna were affected by fish kairomone after three days (3rd instar, Table S1, Fig. 2). With the extension of growth days, the body size of D. magna exposed to fish kairomone was significantly smaller than those not exposed to fish kairomone after five days (5th instar, Table S1, Fig. 2a). The body sizes of $D$. magna exposed or not exposed fish kairomone were close to the maximum after 12 days (9th instar, Table S1, Fig. 2a). Furthermore, With the extension of growth days, the spine length of $D$. magna exposed to fish kairomone was significantly increased than those not exposed to kairomone (Fig. 2d). After 11 days (9th instar, Table S1), the spine length of $D$. magna reached the maximum value regardless of exposure to kairomone, and then began to decrease (Fig. 2d). The spine length / body size of $D$. magna exposed to fish kairomone was significantly higher than that not exposed to kairomone (Fig. 2g). With the extension of growth days, the spine length / body size of D. magna in exposed or not exposed kairomone treatment was close to the minimum after 12 days (9th instar, Table S1, Fig. $2 \mathrm{~g}$ ). 

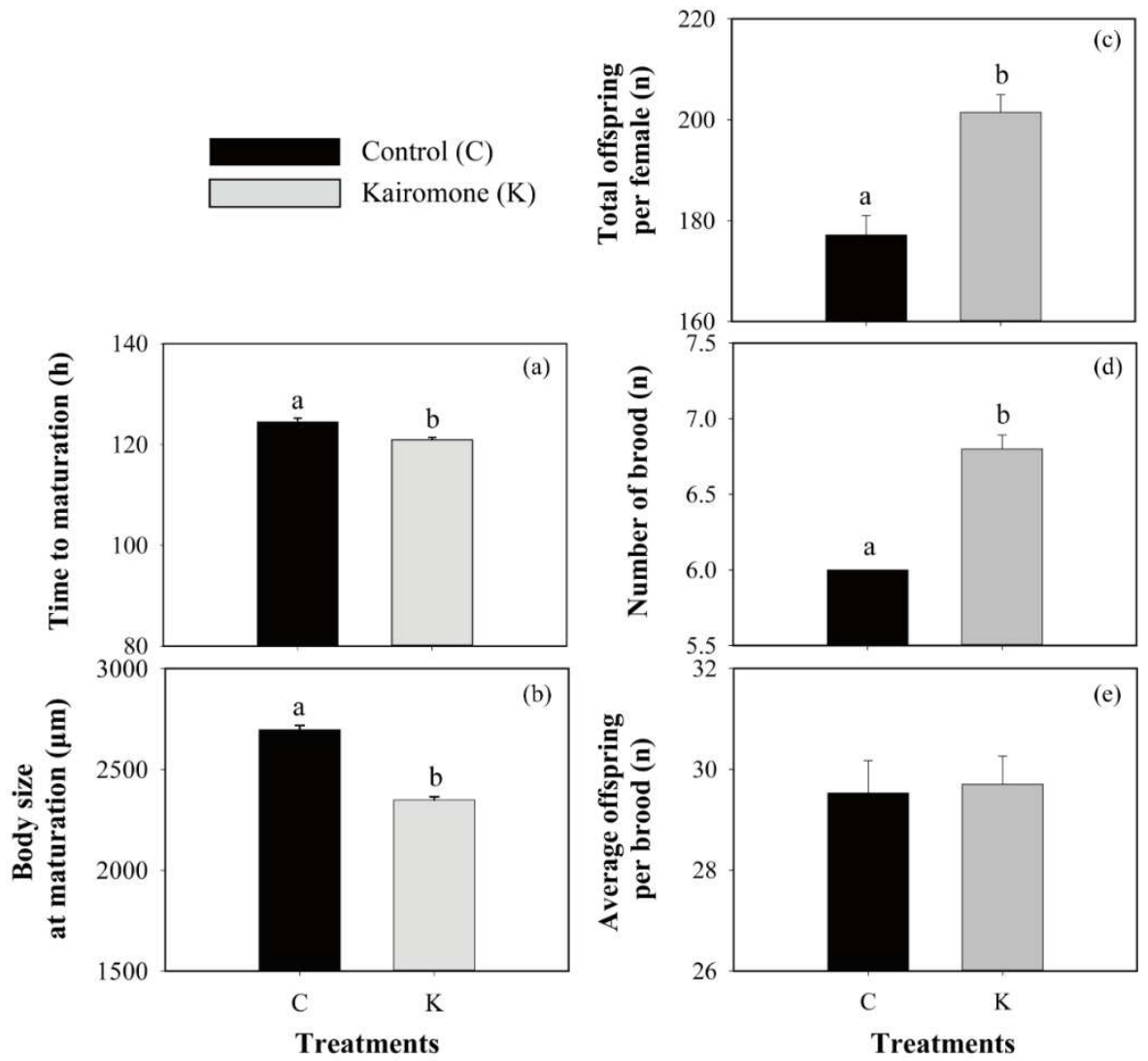

264 Fig. 1 Reproductive traits of D. magna in absence and presence of fish kairomone.

265 The significant differences among different treatments are indicated by the different

266 lowercase letters.

267

268 Table 1 The results of one-way ANOVA on reproductive traits of $D$. magna.

\begin{tabular}{llllll}
\hline Life-history traits & DF & SS & MS & $F$ & $P$ \\
\hline Time to maturation (Fig. 1a) & 1 & 129.600 & 129.600 & 16.482 & $<0.001$ \\
\hline Body size at maturation (Fig. 1b) & 1 & 1167800.59 & 1167800.59 & 160.895 & $<0.001$ \\
\hline Total offspring per female (Fig. 1c) & 1 & 5773.448 & 5773.448 & 21.879 & $<0.001$ \\
\hline Number of brood (Fig. 1d) & 1 & 6.236 & 6.236 & 72.103 & $<0.001$ \\
\hline Average offspring per brood (Fig. 1e) & 1 & 0.306 & 0.306 & 0.0431 & 0.837 \\
\hline
\end{tabular}



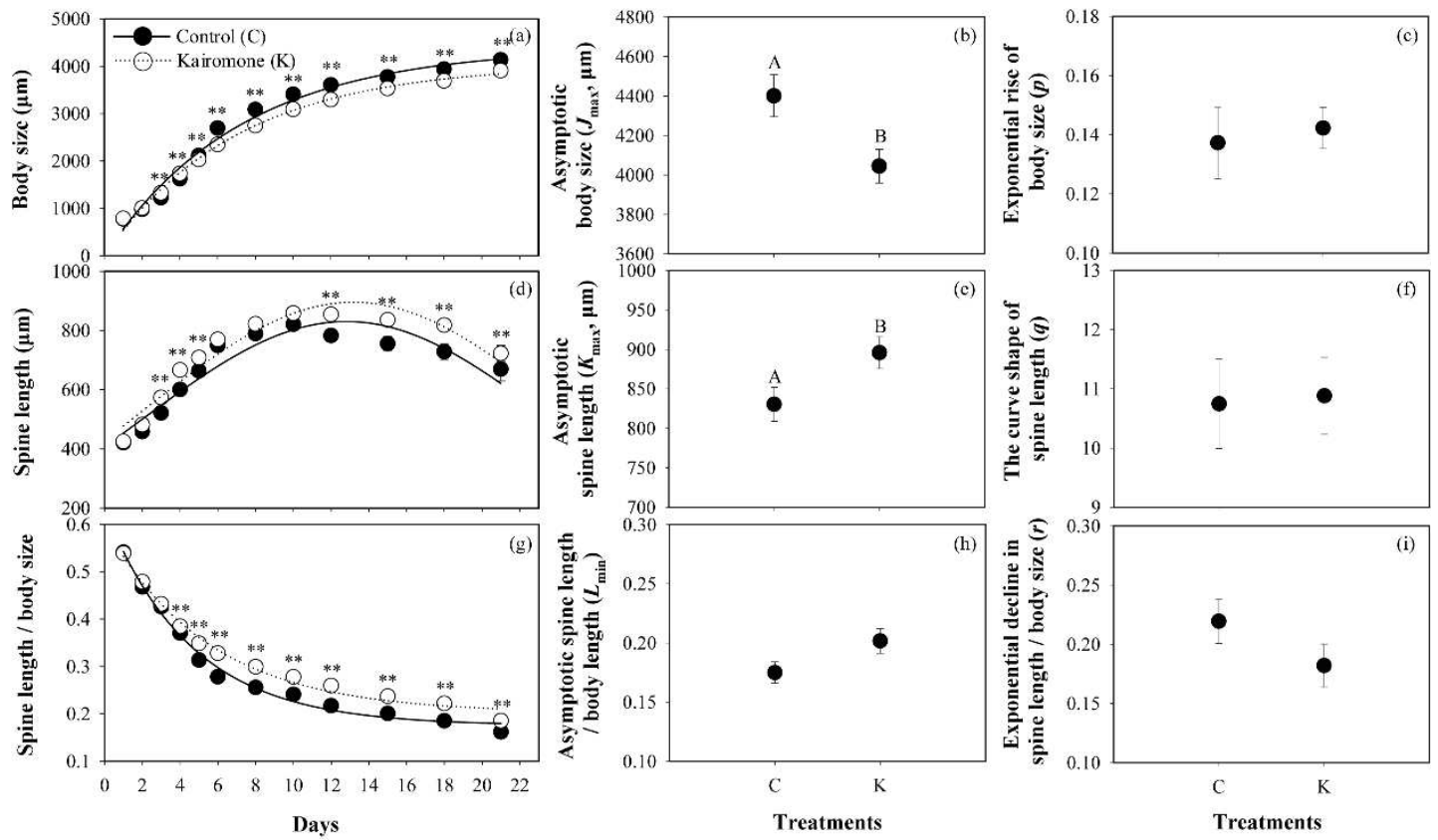

271 Fig. 2 Morphological traits of D. magna in absence and presence of fish kairomone

272 change with days. The asterisks indicate significant differences between the control

273 and fish kairomone treatments under different days $\left({ }^{*} p<0.05, * * p<0.01\right)$. Some

274 error bars are not visible in the figure as they are too short and are covered by the data

275 symbol. The derived parameters (asymptote, the exponential rise rate, and the curve

276 shape) of body size, spine length, and spine length / body size in the control (C) and

277 fish kairomone (K) treatments were compared. The significant differences are

278 indicated by using different uppercase letters.

279

280 Table 2 The results of two-way ANOVA on morphological traits of D. magna.

\begin{tabular}{llllll}
\hline Life-history traits & DF & SS & MS & $F$ & $P$ \\
\hline Body size (Fig. 2a) & & & & & \\
Kairomone & 1 & 2833534.400 & 2833534.400 & 690.170 & $<0.001$ \\
Days & 11 & 568279909.279 & 51661809.934 & 12583.377 & $<0.001$ \\
Kairomone $\times$ days & 11 & 3384809.082 & 307709.917 & 74.950 & $<0.001$ \\
\hline Spine length (Fig. 2d) & & & & & \\
Kairomone & 1 & 263622.719 & 263622.719 & 57.967 & $<0.001$
\end{tabular}




\begin{tabular}{llllll} 
Days & 11 & 8418843.496 & 765349.409 & 168.290 & $<0.001$ \\
Kairomone $\times$ days & 11 & 69952.937 & 6359.358 & 1.398 & 0.170 \\
\hline $\begin{array}{l}\text { Spine length / body size (Fig. 2g) } \\
\text { Kairomone }\end{array}$ & 1 & 0.0883 & 0.0883 & 179.807 & $<0.001$ \\
Days & 11 & 5.548 & 0.504 & 1027.106 & $<0.001$ \\
Kairomone $\times$ days & 11 & 0.0303 & 0.00275 & 5.603 & $<0.001$ \\
\hline
\end{tabular}

\section{Composition of the microbial communities in Daphnia individuals}

283 The family Comamonadaceae (mainly Limnohabitans sp.) showed the highest

284 relative abundance followed by Moraxellaceae (mainly Acinetobacter sp.) and

285 Flavobacteriaceae (mainly Flavobacterium sp.) among all bacterial family (Fig. 3 and

286 S1, Table S2). In the whole Daphnia, there was a significant interaction between

287 kairomone and instar on the relative abundance of Moraxellaceae and

288 Flavobacteriaceae (Table 3), and the relative abundance of Comamonadaceae were

289 significantly decreased by kairomone (Fig. 3, Table 3). In the guts of Daphnia, there

290 was no significant interaction between kairomone and Daphnia instar on the three

291 bacterial family (Table 3). However, as the Daphnia instar increases, the relative

292 abundance of Comamonadaceae and Flavobacteriaceae in the guts were significantly

293 decreased (Fig. 3, Table 3). In the culture medium, there was a significant interaction

294 between kairomone and instar on the relative abundance of the three bacterial family

295 (Table 3); furthermore, the relative abundance of Comamonadaceae was significantly

296 reduced under kairomone influence (Fig. 3, Table 3). 


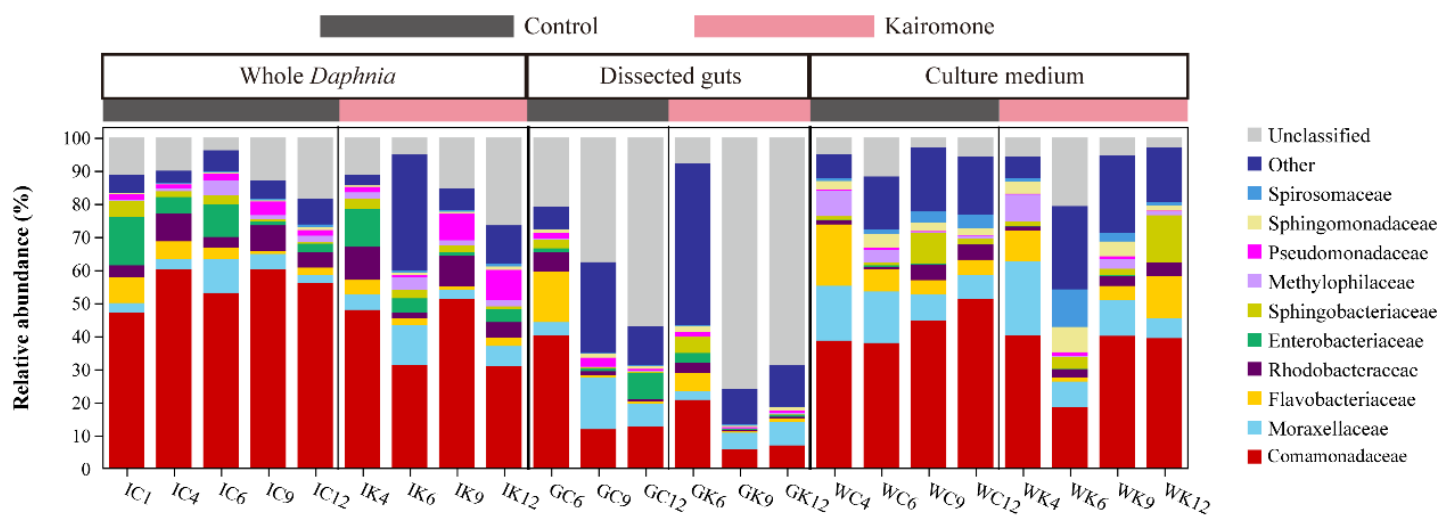

Groups

299 Fig. 3 Relative abundance of bacterial at the family level. The left, middle, and right sections represent whole Daphnia (left), dissected guts (middle), and culture medium (right) of D. magna with different instars in absence and presence of fish kairomone, respectively. The numbers "1", “4”, “6", “9” and "12" represent different instars.

Table 3 The results of two-way ANOVA on the relative abundance of bacterial.

\begin{tabular}{|c|c|c|c|}
\hline Site of microflora detection & $\begin{array}{l}\text { Bacterial specie (family } \\
\text { level) }\end{array}$ & $F$ & $P$ \\
\hline \multirow[t]{12}{*}{ Whole Daphnia } & Comamonadaceae & & \\
\hline & Kairomone & 9.276 & 0.006 \\
\hline & instar & 0.227 & 0.920 \\
\hline & Kairomone $\times$ instar & 1.667 & 0.197 \\
\hline & Moraxellaceae & & \\
\hline & Kairomone & 1.907 & 0.182 \\
\hline & instar & 1.249 & 0.323 \\
\hline & Kairomone $\times$ instar & 4.818 & 0.007 \\
\hline & Flavobacteriaceae & & \\
\hline & Kairomone & 0.842 & 0.370 \\
\hline & instar & 11.689 & $<0.001$ \\
\hline & Kairomone $\times$ instar & 16.893 & $<0.001$ \\
\hline \multirow[t]{7}{*}{ Dissected guts } & Comamonadaceae & & \\
\hline & Kairomone & 2.630 & 0.156 \\
\hline & Days & 15.852 & 0.004 \\
\hline & Kairomone $\times$ days & 0.0811 & 0.923 \\
\hline & Moraxellaceae & & \\
\hline & Kairomone & 1.911 & 0.216 \\
\hline & Days & 2.035 & 0.211 \\
\hline
\end{tabular}




\begin{tabular}{|c|c|c|c|}
\hline & Kairomone $\times$ days & 1.412 & 0.314 \\
\hline & Flavobacteriaceae & & \\
\hline & Kairomone & 3.274 & 0.120 \\
\hline & Days & 13.543 & 0.006 \\
\hline & Kairomone $\times$ days & 3.618 & 0.093 \\
\hline Culture medium & Comamonadaceae & & \\
\hline & Kairomone & 19.169 & $<0.001$ \\
\hline & Days & 14.565 & $<0.001$ \\
\hline & Kairomone $\times$ days & 5.320 & 0.010 \\
\hline & Moraxellaceae & & \\
\hline & Kairomone & 0.00421 & 0.949 \\
\hline & Days & 14.690 & $<0.001$ \\
\hline & Kairomone $\times$ days & 4.190 & 0.023 \\
\hline & Flavobacteriaceae & & \\
\hline & Kairomone & 2.501 & 0.133 \\
\hline & Days & 22.109 & $<0.001$ \\
\hline & Kairomone $\times$ days & 14.185 & $<0.001$ \\
\hline
\end{tabular}

\section{Diversity analysis in the microbiome}

Alpha diversity, such as Shannon (Fig. 4a), of microbiota in dissected guts and whole Daphnia did not change significantly with the increase of Daphnia instar. However, in the presence of fish kairomone, the interaction with Daphnia instar $(P=$ 0.008) significantly reduced the Shannon diversity of microbiota in dissected guts (Fig. 4a). The interaction between kairomone and Daphnia instar was not observed in Shannon diversity of whole Daphnia microbiota. We also detected the Shannon diversity of microbiota in the culture medium, and found that the increase of Daphnia instar did not change the Shannon diversity of microbiota in the culture medium, but the interaction between kairomone and Daphnia instar had a significantly effect on the Shannon diversity of microbiota in the culture medium $(P=0.005$, Fig. 4a). 
(a)

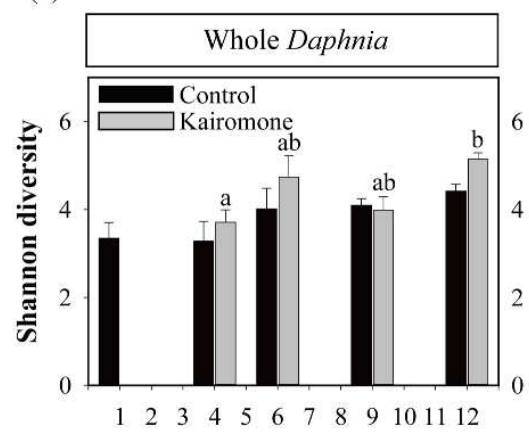

(b)

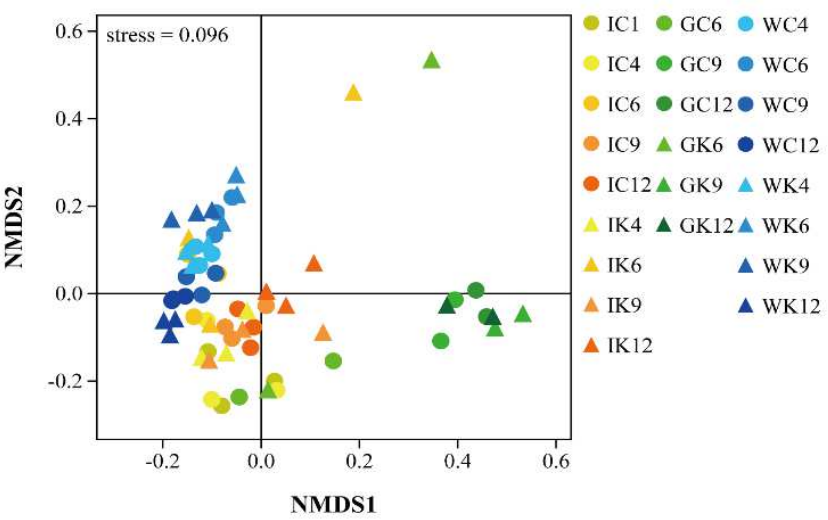

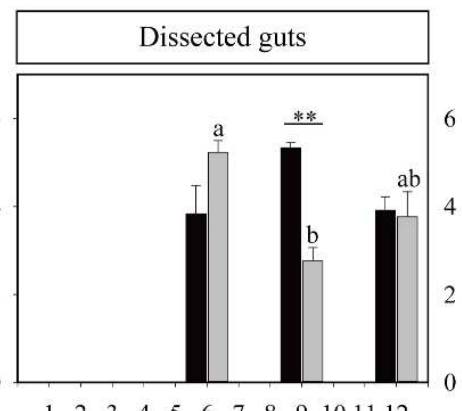

$\begin{array}{lllllllllllll}1 & 2 & 3 & 4 & 5 & 6 & 7 & 8 & 9 & 10 & 11 & 12\end{array}$

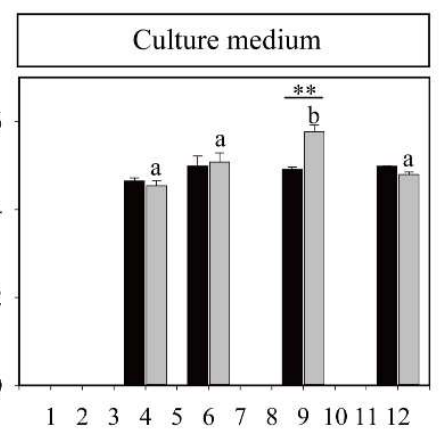

(c)

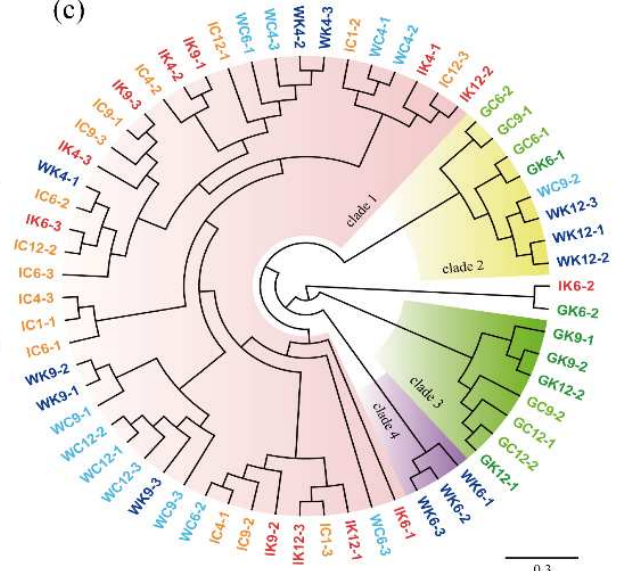

Fig. 4 Changes of alpha and beta diversity in D. magna bacterial communities. (a)

Shannon diversity in whole Daphnia (left), dissected guts (middle), and culture medium (right) of D. magna in absence and presence of fish kairomone with different instars. Bars indicate mean values, and error bars indicate the standard error. The dark bars with asterisks indicate significant differences between the control and fish kairomone treatments under different instars $(* \mathrm{p}<0.05, * * \mathrm{p}<0.01)$. The significant 
differences among different instars are indicated by the different uppercase (for the control) or lowercase (for the kairomone treatment) letters. (b) Two-dimensional nonmetric multidimensional scaling (NMDS) plot of bacterial communities. Bray-Curtis distance matrix was used to generate the NMDS plots. "I" means "whole Daphnia" (red gradient), "G" means "dissected guts" (green gradient), and "W" means "culture medium" (blue gradient). The "C" (circle) and "K" (triangle) represent the control and fish kairomone treatments, respectively. (c) Unweighted pair group method with arithmetic mean (UPGMA) tree of bacterial communities. Bray-Curtis distance matrix was used to generate the UPGMA tree. "I" means "whole Daphnia" (red gradient), "G" means "dissected guts" (green gradient), and "W" means "culture medium" (blue gradient). The letters " $\mathrm{C}$ " and "K" represent the control and fish kairomone treatments, respectively.

\section{Representative OTUs}

There were small overlaps in OTUs of microbiota within whole Daphnia, dissected guts, and culture medium, while most OTUs in each part were unique (Fig. 5a). In the gut microbiota, OTUs between absence and presence of fish kairomone also overlapped small, and the number of unique OTUs was greater among the control and fish kairomone treatments (Fig. 5b). By detailed profiling of the OTU dynamics in the control and fish kairomone treatments, we found 9 OTUs belonged to, for example, Arcobacter and Helicobacter in Epsilonbacteraeota, that were found only in the gut microbiota exposed to fish kairomone. In addition, 295 OTUs belonging to 
phylum Firmicutes were found in the gut microbiota exposed to fish kairomone.

However, there were only 217 OTUs belonging to phylum Firmicutes found in the gut microbiota not exposed to fish kairomone. It is worth noting that the sum of these OTUs only accounted for a small proportion of the total relative abundance of all phyla (Additional file 2).

(a)

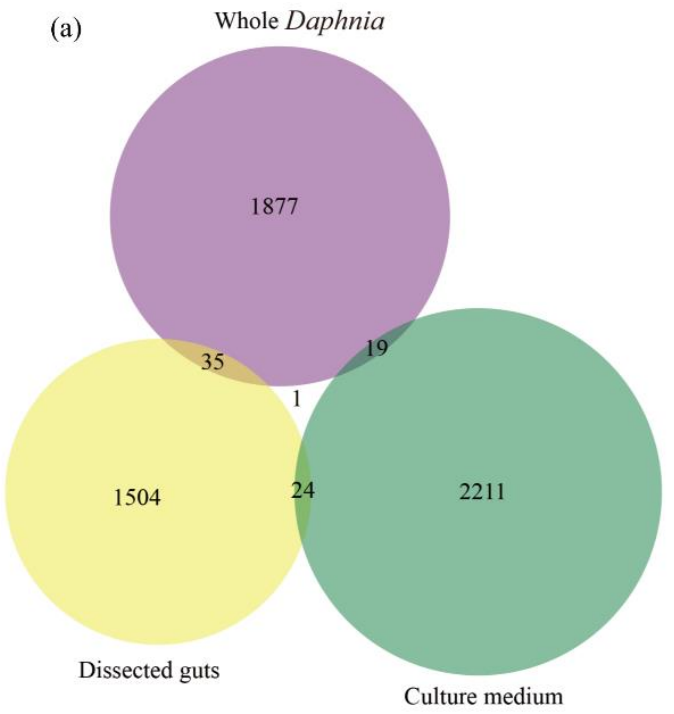

(b)

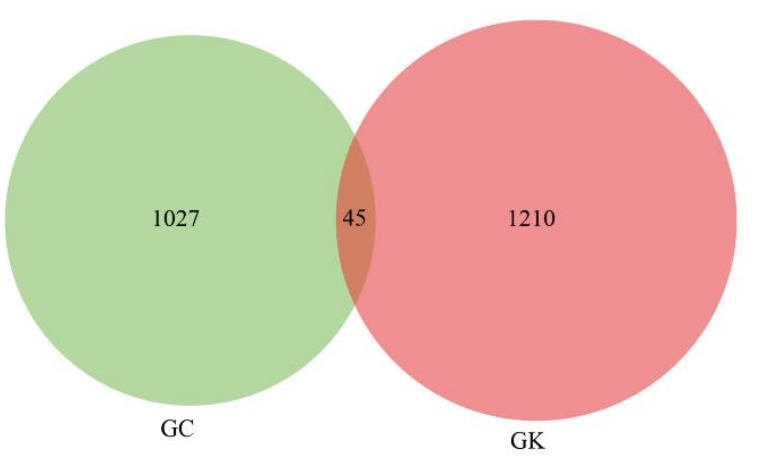

Fig. 5 Profiling of OTUs in D. magna microbial communities. Venn diagrams showing (a) number of OTUs in different parts, (b) number of OTUs in control (C) (green) and fish kairomone (K) (red) treatments in dissected guts.

\section{Correlation between life history traits and bacterial abundance}

The body size and the spine length of D. magna were significantly correlated with the relative abundance of Comamonadaceae in both whole Daphnia and culture medium microbiota (Fig. 6, Table S3). The body size and the spine length of $D$. magna increased and decreased by the high relative abundance of Comamonadaceae, 
respectively (Fig. 6, Table S3). In the whole Daphnia, the relative abundance of other bacteria (Moraxellaceae and Flavobacteriaceae) were not correlated with both body size and spine length of D. magna (Fig. 6, Table S3). In the culture medium, the spines length of $D$. magna was also reduced by the high relative abundance of Flavobacteriaceae (Fig. 6, Table S3). In dissected guts of Daphnia, except that the spines length of $D$. magna was reduced by the high relative abundance of Flavobacteriaceae (Fig. 6, Table S3), the relative abundance of the three bacterium showed no correlation with the body size and spine length D. magna (Fig. 6, Table S3).

D. magna key life-history traits, such as the total offspring per female and the number of brood, were directly linked with the relative abundance of Comamonadaceae and Moraxellaceae in the whole Daphnia (Fig. 6, Table S3). The two traits (total offspring per female and number of brood) of D. magna were decreased by the high abundance of Comamonadaceae and increased by the high abundance of Moraxellaceae (Fig. 6, Table S3). In the culture medium, only the total offspring per female of D. magna was reduced by the high abundance of Comamonadaceae (Fig. 6, Table S3). In dissected guts of Daphnia, the abundance of the three bacterium showed no correlation with the two parameters (total offspring per female and number of brood) of D. magna (Fig. 6, Table S3). 


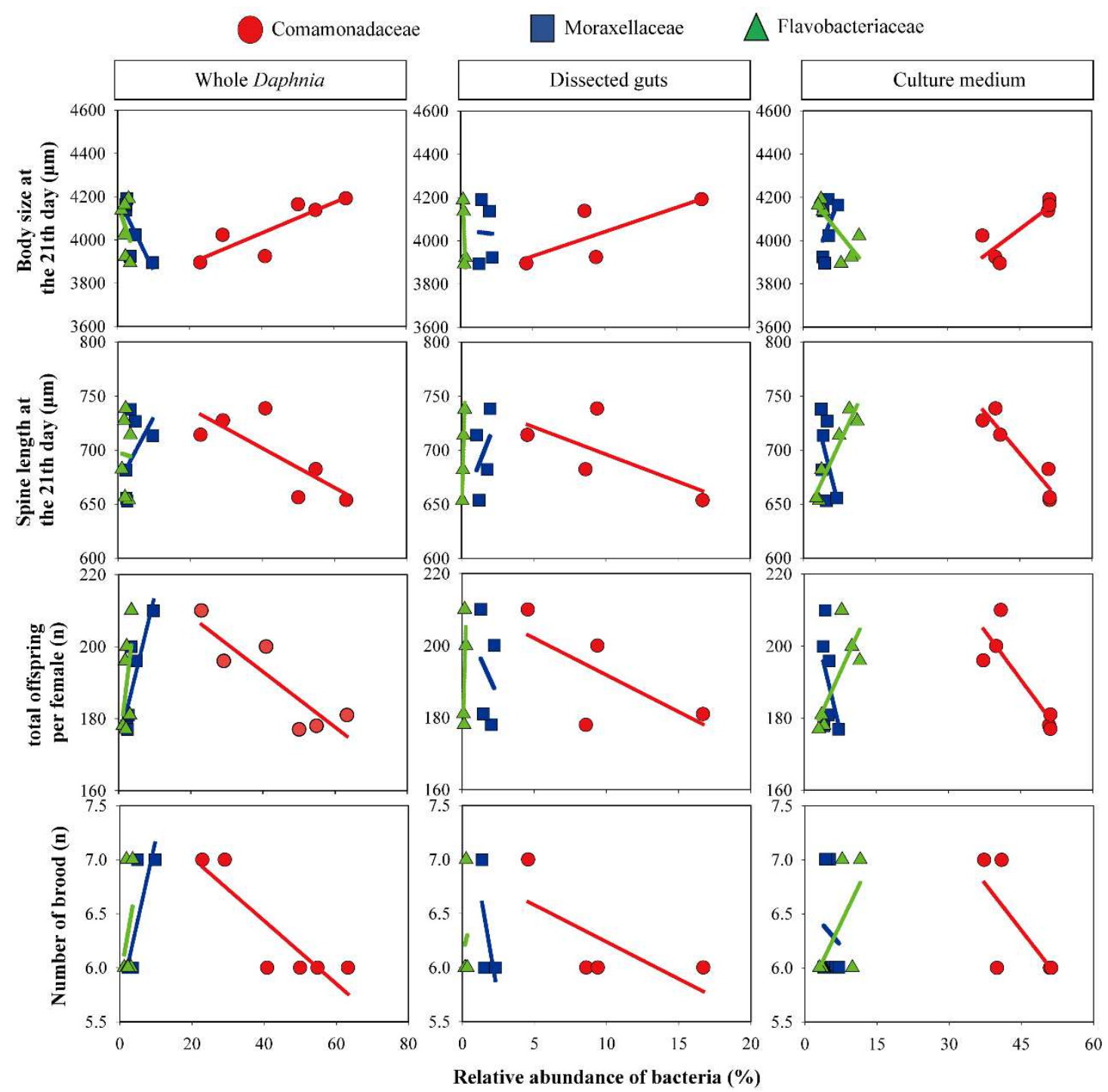

Fig. 6 Scatterplot of correlation between key life-history traits and family level

392 abundances of bacteria. The data were fitted by linear regression model. The left, middle, and right sections represent whole Daphnia (left), dissected guts (middle),

394 and culture medium (right) of D. magna, respectively. When the slope was

395 significantly different from zero $(\alpha=0.05)$, this indicated a significant correlation.

$396 \quad$ Values of $R^{2}$ and $P$ are shown in Table S3. 

showed significantly negative correlation with the microbial diversity detected in

402 culture medium (Fig. 7, Table S4). For the total offspring per female and the brood

403 number of $D$. magna, only the brood number was found positive correlation with the microbial diversity detected in the whole Daphnia (Fig. 7, Table S4). The body size and the total offspring number of D. magna had no significant correlation with microbial diversity detected in the three microflora detection sites (Fig. 7, Table S4).
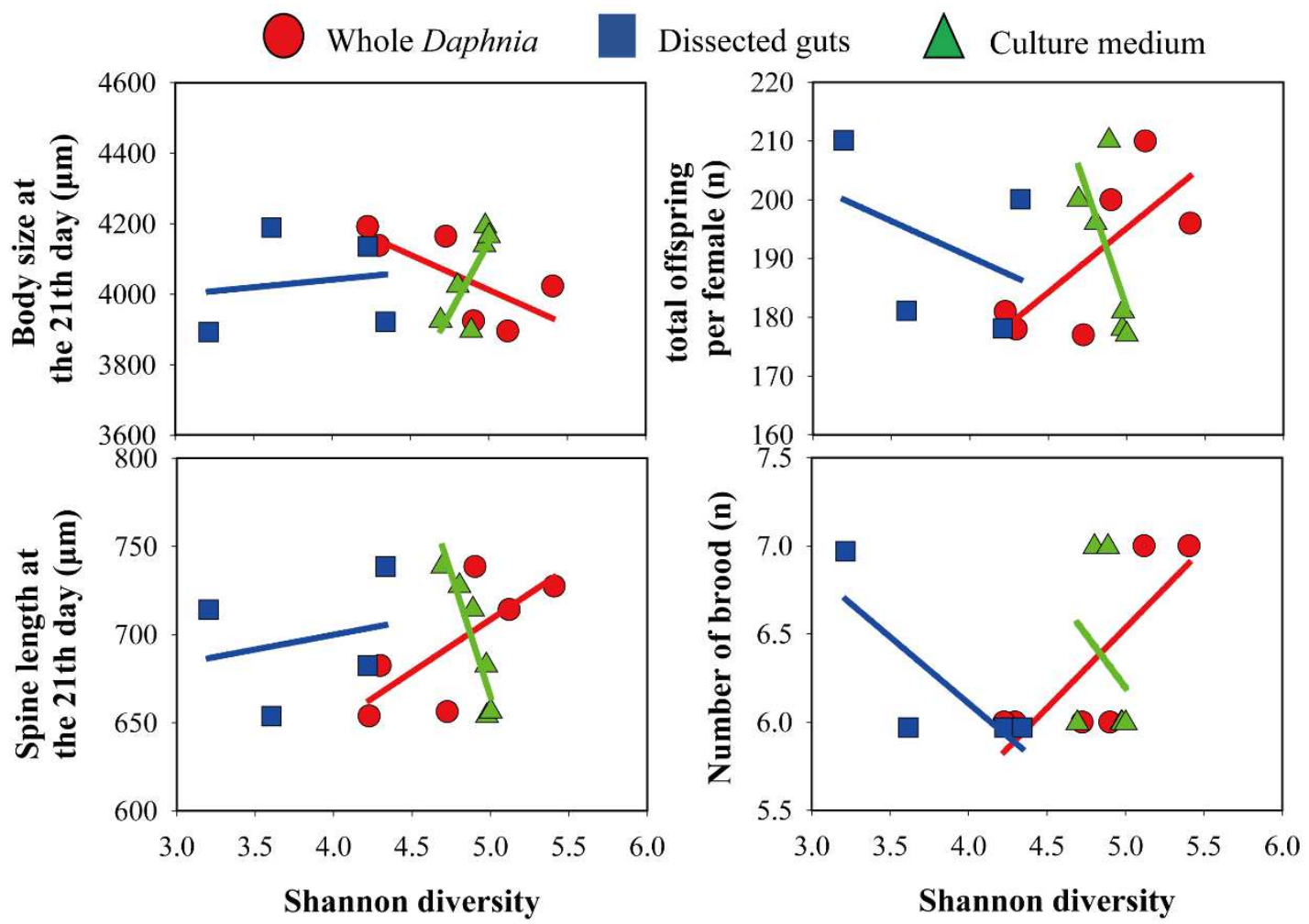

Fig. 7 Scatterplot of correlation between key life-history traits and microbial diversity in different microflora detection sites. The data were fitted by linear regression model.

411 When the slope was significantly different from zero $(\alpha=0.05)$, this indicated a significant correlation. Values of $R^{2}$ and $P$ are shown in Table S4. 
PICRUSt2 gave 36 predicted functional categories that represented 7 pathway maps in the KEGG level 2 functional modules. Multiple KEGG function abundance

417 cluster analysis was conducted to check the changes of functions under the absence and presence of fish kairomone and different microflora detection sites (Fig. 8). When analyzing the effects of fish kairomone on microbial functional categories in whole Daphnia and culture medium, we found that there was little difference between different functional categories in whole Daphnia and culture medium in the presence or absence of fish pheromone (Fig. 8). However, when analyzing the effects of fish kairomone on microbial functional categories in dissected guts, we found that there was a significant effect on metabolic pathways, involved in enhancing the metabolism of cofactors, vitamins, and energy (Fig. 8). Furthermore, the presence of fish kairomone enhanced the functional categories folding, sorting, and degradation (Fig. 8). 


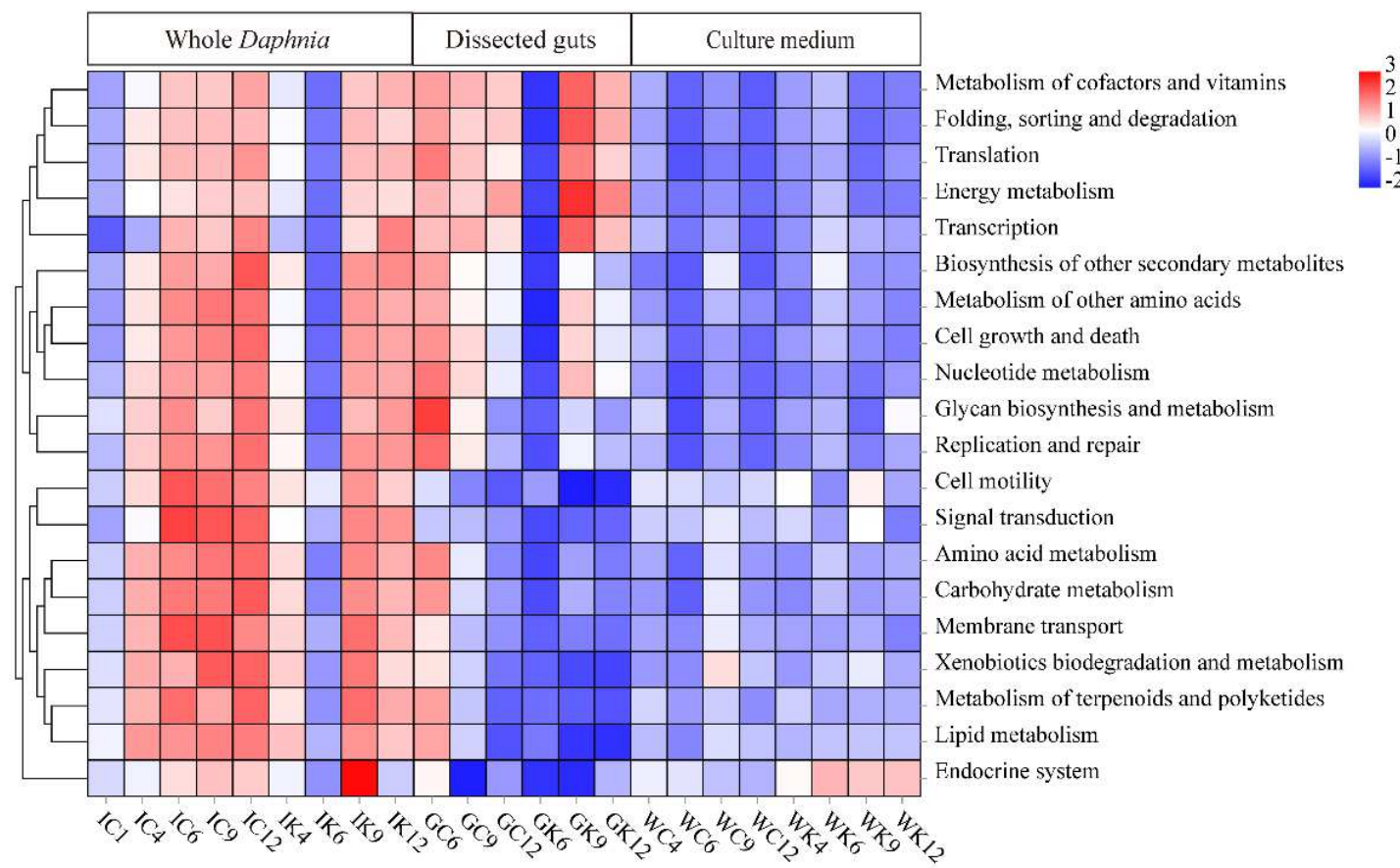

Fig. 8 Relative abundance of each predicted functional categories given in KEGG pathways (level 2). The left, middle, and right sections represent whole Daphnia (left), dissected guts (middle), and culture medium (right) of D. magna in absence and presence of fish kairomone, respectively. The numbers "1", “4", "6", "9" and " 12 " represent different instars.

\section{Discussion}

Over the past few years, evidences are accumulating that the gut microbiota can be a crucial mediator of life history variation, as well as of acclimatization and adaptation to changing environmental conditions $[8,9]$. Predicting how, and to what extent, the gut microbiota may impact fitness requires to identify the links between variation in gut microbiota and host phenotype, and to understand how the microbiome communities are assembled [8]. In this study, we analyzed the 
to D. magna under the action of fish kairomone. As we hypothesized, with the increase of instars, fish kairomone changes the gut microbiota composition and decreases the $\alpha$ diversity of $D$. magna gut microbiota. The relative abundance of some bacteria decreases due to the presence of kairomone, among which,

Comamonadaceae (mainly Limnohabitans sp.) is mainly reduced. Correlation analysis results showed that there was a linear correlation between the high relative abundance of Comamonadaceae, Moraxellaceae, and Flavobacteriaceae and the life history traits of $D$. magna. Among them, the body size of $D$. magna was positively correlated with the increased abundance of Comamonadaceae, while spine length of D. magna was negatively correlated with the increased abundance of Comamonadaceae, and positively correlated with the increased abundance of Flavobacteriaceae. Predation risk can affect the composition of gut microbiota during the growth $D$. magna, which may indirectly affect the defensive traits of $D$. magna. This result is of great significance for understanding the influence of host-microbial interaction on individual anti-predation defense.

\section{D. magna gut microbiota}

Peter and Sommaruga [49] detected most major bacterial groups of the surrounding water in gut homogenates of copepods and cladocerans, except for Actinobacteria. Thus, these different community members which were accumulated in the gut through filtration could reflect bacteria from the cultivation water. However, in our study, D. magna has a unique gut microbiota, and there were strong differences between microbial communities in the Daphnia gut and in the surrounding water. 
Grossart et al. [50] demonstrated that bacteria associated with the cladoceran Bosmina still remained highly similar when transplanted to another lake. Thus, it can be assumed that the gut microbiota community in Daphnia is composed, at least to a major part, of resident bacteria and is less susceptible to reflect the surrounding bacterial community. In agreement with previous reports $[5,13,51,52]$, Proteobacteria and Bacteroidetes were the dominant bacterial phyla in D. magna guts in our study. Among them, Proteobacteria is dominant, and Comamonadaceae (mainly Limnohabitans sp.) is the main component of Proteobacteria phyla. Comamonadaceae family induced positive fitness effects in Daphnia, and Limnohabitans was the most abundant Comamonadaceae, as was described in previous studies [27, 53-56]. In this study, with the increase of Daphnia instar, the abundance of Comamonadaceae (mainly Limnohabitans sp.) in Daphnia guts was significantly reduced (Table 3). It was observed that the microbiota community composition did not remain stable over time. However, fish kairomone had no significant effect on the relative abundance of Comamonadaceae (mainly Limnohabitans sp.) in Daphnia guts, nor did the interaction with instars, although fish kairomones significantly increased the fecundity of D. magna (Fig. 1 and 2, Table 3). This seems to contradict the results of Peerakietkhajorn et al. [56, 57], which found bacteria in the genus Limnohabitans have been linked to increased fecundity and population size in D. magna. Possibly, this shift in the community composition is caused by two reasons. For one thing, Daphnia might spend costs on growth in the early stage, and turn to spend more energy on resisting predators in the later 
developmental stage as time goes on. For another, Daphnia might uptake and stimulate the inactive or underrepresented bacteria, as described in the guts of earthworms [58]. These bacteria were not detected, but were activated and thus increased in abundance over time, overriding the relative abundance of Limnohabitans. In addition to Limnohabitans, other bacteria detected in D. magna intestine include Acinetobacter and Flavobacterium. Furthermore, when comparing the relative abundance of bacterial phyla among treatments, we found that Epsilonbacteraeota only existed in the gut microbiota of D. magna exposed to fish kairomone. This suggests that Epsilonbacteraeota, especially Arcobacter and Helicobacter could be used as indicators for predation risk. In addition, Epsilonbacteraeota have also been suggested to be associated with hypertension [59], and the increased risk for hypertension is often associated with host psychological stress $[60,61]$.

\section{Effects of predation risk and Daphnia instar on D. magna gut microbiota}

In previous studies on humans and some vertebrates, researchers have shown that stress can affect and alter the gut microbiota. For example, the fecal lactic acid bacterial levels decreased significantly when students were facing academic stress [62]; social disruption stressor could impact the gut microbiota community in mice [63]; in aquatic organisms, the species richness and microbial diversity of overall gut microbiota in perch significantly decreased with predator presence [12]. In our experiment, the presence of predation risk interfered with the diversity of D. magna gut microbiota, and the diversity of gut microbiota also significantly decreased with 

are influenced by species richness and composition [64], more diverse communities possibly contain a wider array of metabolic capabilities. However, coping with stress is a process of expending energy for animals and may have an impact on metabolism. For example, the haemoglobin concentration in the tissues of Daphnia pulex decreased in the presence of kairomones [65]. A lower concentration of haemoglobin could be responsible for a decreased efficiency of oxygen transport to body tissues, which in turn leads to a decrease in energy for growth and reproduction [65]. In this study, the presence of fish kairomone also affected the metabolic pathway of $D$. magna (Fig. 8). Thus, animals might need to re-allocate metabolic substrates to other

tissues to cope with the increasing energy needs when facing stress, such as to stimulate oxygen in gills [66], instead of spending them on a high-energy intestine [67]. Alternately, there are differences in the composition of the gut microbiota community at different Daphnia instars, and these differences may be due to the physiological changes that occur during development of Daphnia. For example, in contrast to the small body size of Daphnia juvenile, the increased body size after juvenile growth could result in longer gut passage time and improved assimilation efficiency [68]. Furthermore, the characteristics of transportation time and morphological structure in the digestive system will affect the community composition of gut microbiota. Longer gut passage time may make the gut microbiota community have a longer time to use substrates $[69,70]$. This may be the main reason that Daphnia instar interacts with fish kairomone and significantly reduces the gut 
microbiota diversity of D. magna.

\section{Relationship between gut bacteria and D. magna life history traits}

In the study on the comparing germ-free and conventionally reared individuals of D. magna, Sison et al. [4] first reported that the gut microbiota is an important factor that affects life-history traits contributing to host fitness. Compared with conventionally reared individuals, germ-free D. magna have smaller body size, less fecundity, and higher mortality [4]. In the food chain, the body size and reproduction quantity of prey are important traits to cope with predation pressure $[41,71,72]$. For Daphnia, the significant reduction in body size and increase in fecundity can allow them to increase the probability of reproduction before being eaten by visual, sizeselective predators such as fish [71-73]. Current study also shows that the presence of fish kairomone significantly decreased the body size and increased the spine length, the total offspring number of D. magna (Fig. 1 and 2, Table 1 and 2). We chose these traits as representative parameters to establish the correlation between the abundance and composition of gut microbiota and D. magna fitness, which were consist with previous studies that used several key features such as fecundity, host survival time, and body size to correlate host fitness with the microbiome $[13,51,74,75]$. In our study, the reproductive traits (the total offspring number and the brood number) of $D$. magna were directly linked with the abundance of either Comamonadaceae or Moraxellaceae; the growth traits (the body size and the spine length) of $D$. magna were directly linked with Comamonadaceae abundance (Fig. 6, Table S3). Previous studies have shown that some strains of bacteria can provide the essential elements for 
host reproduction and growth to the benefit of the host $[57,75]$. For example, the key components of Daphnia gut microbiota, Limnohabitans, Aeromonas, and Acidovorax $[56,76]$, have been linked to Daphnia obtaining essential amino acids $[77,78]$, polyunsaturated fatty acids, and sterols [79] that positively affect Daphnia growth and reproduction [78]. More specifically, these bacteria in Daphnia's gut can produce some useful enzymes for digestion [57], and then increase the production of nutrients incorporated into the female and parthenogenetic eggs during development of oocytes, which may promote Daphnia growth and increase numbers of viable Daphnia juveniles. In our results, the presence of fish kairomone significantly decreased the relative abundance of Comamonadaceae (mainly Limnohabitans sp.), which could be the main reason for the body size reduction of D. magna. However, the reproduction quantity of D. magna increased significantly in the presence of fish kairomone, which may depend on the abundance and presence of Moraxellaceae (Gammaproteobacteria, Pseudomonadales). Although the knowledge of Moraxellaceae function in freshwater invertebrates is rarely, but some genus in Pseudomonadales, e.g., Pseudomonas sp., can widely colonize in Daphnia and have a positive effect on Daphnia growth and survival [80]. We speculated that Moraxellaceae plays the same role as Comamonadaceae in improving the fecundity of Daphnia, and Moraxellaceae is more competitive than Comamonadaceae in the presence of fish kairomone. Moreover, the host growth and reproduction may also acquire essential nutrients from more available pathways. It should be noted that the correlation in our results was more pronounced in 
whole Daphnia and culture medium microorganisms, but not in Daphnia gut, which may be because Daphnia feeding behavior plays an important role in structuring Daphnia-associated microbial communities [55, 81]. For shaping the gut environment, host metabolism and immunity might further extend to influence the external environment of Daphnia, for instance through the shedding of immune effectors [82], thus affecting the bacterioplankton community structure. This is in accordance with the results of Mack et al. [83], who found that diet or microbial inoculation has less influence on the gut microbiota, while has greater influence on the surrounding environmental microbiota.

\section{Conclusions}

In this study, we found that D. magna not only developed defense traits, but also changed gut microbiota in response to predation risk. The change in the abundance of microbiota was mainly manifested in the abundance of Comamonadaceae which was significantly reduced under the influence of fish kairomone. As for defense traits, the body size of D. magna was positively correlated with the increased abundance of Comamonadaceae. The spine, total offspring number, and brood number of D. magna were negatively correlated with the increased abundance of Comamonadaceae. In addition, the co-action of Daphnia instar and fish kairomone significantly reduced the diversity of gut microbiota, which may be related to the high diversity of gut microbiota that may consume more Daphnia resources which are necessary for its growth. This study suggests that gut microbiota play an important role in the 
development of defensive traits in Daphnia in response to fish predators. Linking defense characteristics to the change of microbiota may help us to better predict hostmicrobiota interactions under multiple stressors, and further analysis is needed to better understand these relationships.

\section{Abbreviations}

ANOVA: Analysis of variance; NMDS: Non-metric multidimensional scaling;

UPGMA: Unweighted pair group method with arithmetic; C: Absence of fish

kairomone; K: Presence of fish kairomone; Site of microflora detection-I: Whole

Daphnia; Site of microflora detection-G: Dissected guts; Site of microflora detection-

W: Culture medium.

\section{Supplementary Information}

Additional file 1: Table S1. Measurement time and corresponding instar of $D$. magna. Table S2. List of bacteria genus found in the microbiome of D. magna. Table

S3. Correlation between different traits and different dominant bacterial groups. Table S4. Correlation between different traits and microbial diversity in different microflora detection sites. Fig. S1. Relative abundance of bacteria at the different level of $D$. magna. The left, middle, and right sections represent whole Daphnia (left), dissected guts (middle), and culture medium (right) of D. magna with different instars in absence and presence of fish kairomone, respectively. The numbers "1", "4", “6”, "9" and " 12 " represent different instars. 
623 Not applicable.

Not applicable.

The data of raw sequences for microbial sequence data were deposited in...

\section{Competing interests}

632 The authors declare that they have no known competing financial interests or personal 633 relationships that could have appeared to influence the work reported in this paper.

\section{Funding}

636 This research was supported by the Key Project of National Natural Science

637 Foundation of China (31730105) and the Priority Academic Program Development of 638 Jiangsu Higher Education Institutions. 
641 All authors read and approved the final manuscript for submission. QL and ZY

642 designed the experiments. QL, SA, QMZ, and ZHD conducted the experiments and

643 collected the data. QL, YFS, and ZHD analyzed the data. QL, LG, and SA wrote the

644 initial draft of the manuscript, while ZY provided substantial feedback.

645

646 Acknowledgements

647 We greatly appreciate the anonymous reviewers for their constructive comments.

648

649

Authors' information

650

Jiangsu Key Laboratory for Biodiversity and Biotechnology, School of Biological

651 Sciences, Nanjing Normal University, 1 Wenyuan Road, Nanjing 210023, China.

652

653

\section{References}

654 [1] Douglas AE. Multiorganismal insects: diversity and function of resident

655 microorganisms. Annu Rev Entomol. 2015; 60: 17-34.

656 https://doi.org/10.1146/annurev-ento-010814-020822.

657 [2] Amato KR. Incorporating the gut microbiota into models of human and non-

658 human primate ecology and evolution. Am. J. Phys. Anthropol. 2016; 159: 196-215.

659 https://doi.org/10.1002/ajpa.22908.

660 [3] Engel P, Moran NA. The gut microbiota of insects - diversity in structure and

661 function. FEMS Microbiol. Rev. 2013; 37(5): 699-735. https://doi.org/10.1111/1574-

662

$\underline{6976.12025 .}$. 
663

664

665

666

667

668

669

670

671

672

673

674

675

676

677

678

679

680

681

682

683

684

[4] Sison-Mangus MP, Mushegian AA, Ebert D. Water fleas require microbiota for

survival, growth and reproduction. ISME J. 2015; 9(1): 59-67.

https://doi.org/10.1038/ismej.2014.116.

[5] Callens M, Macke E, Muylaert K, Bossier P, Lievens B, Waud M, et al. Food availability affects the strength of mutualistic host-microbiota interactions in Daphnia magna. ISME J. 2016; 10(4): 911-20. https://doi.org/10.1038/ismej.2015.166.

[6] Storelli G, Defaye A, Erkosar B, Hols P, Royet J, Leulier F. Lactobacillus plantarum promotes drosophila systemic growth by modulating hormonal signals through TOR-dependent nutrient sensing. Cell Metab. 2011; 14(3): 403-14.

https://doi.org/10.1016/j.cmet.2011.07.012.

[7] Coon KL, Vogel KJ, Brown MR, Strand MR. Mosquitoes rely on their gut microbiota for development. Mol. Ecol. 2014; 23(11): 2727-39.

https://doi.org/10.1111/mec.12771.

[8] Alberdi A, Aizpurua O, Bohmann K, Zepeda-Mendoza ML, Gilbert MTP. Do vertebrate gut metagenomes confer rapid ecological adaptation? Trends Ecol. Evol. 2016; 31(9): 689-99. https://doi.org/10.1016/j.tree.2016.06.008.

[9] Macke E, Tasiemski A, Massol F, Callens M, Decaestecker E. Life history and eco-evolutionary dynamics in light of the gut microbiota. Oikos. 2017; 126(4): 50831. https://doi.org/10.1111/oik.03900.

[10] Shapira M. Gut microbiotas and host evolution: scaling up symbiosis. Trends Ecol. Evol. 2016; 31(7): 539-49. https://doi.org/10.1016/j.tree.2016.03.006.

[11] Sullam KE, Essinger SD, Lozupone CA, O'connor MP, Rosen GL, Knight R, et 
685

686

687

688

689

690

691

692

693

694

695

696

697

698

699

700

701

702

703

704

705

706

al. Environmental and ecological factors that shape the gut bacterial communities of fish: a meta-analysis. Mol. Ecol. 2012; 21(13): 3363-78.

\section{https://doi.org/10.1111/j.1365-294x.2012.05552.x.}

[12] Zha Y, Eiler A, Johansson F, Svanback R. Effects of predation stress and food ration on perch gut microbiota. Microbiome. 2018; 6(1): 28.

https://doi.org/10.1186/s40168-018-0400-0.

[13] Akbar S, Gu L, Sun Y, Zhou Q, Zhang L, Lyu K, et al. Changes in the life history traits of Daphnia magna are associated with the gut microbiota composition shaped by diet and antibiotics. Sci. Total Environ. 2020; 705: 135827.

\section{https://doi.org/10.1016/j.scitotenv.2019.135827.}

[14] Svanbäck R, Persson L. Individual diet specialization, niche width and population dynamics: implications for trophic polymorphisms. J. Anim. Ecol. 2004; (73): 973-82. https://doi.org/10.1111/j.0021-8790.2004.00868.x.

[15] Svanbäck R, Eklöv P, Fransson R, Holmgren K. Intraspecific competition drives multiple species resource polymorphism in fish communities. Oikos. 2008; 117(1):

114-24. https://doi.org/10.1111/j.2007.0030-1299.16267.x.

[16] Tonn WM, Holopainen IJ, Paszkowski CA. Density-dependent effects and the regulation of crucian carp populations in single-species ponds. Ecology. 1994; (75): 824-34. https://doi.org/10.2307/1941738.

[17] Brönmark C, Miner JG. Predator-induced phenotypical change in body morphology in crucian carp. Science. 1992; (258): 1348-50.

https://doi.org/10.1126/science.258.5086.1348. 
[18] Johansson F, Andersson J. Scared fish get lazy, and lazy fish get fat. J. Anim. Ecol. 2009; 78(4): 772-7. https://doi.org/10.1111/j.1365-2656.2009.01530.x.

[19] Svanbäck R, Zha Y, Brönmark C, Johansson F. The interaction between predation risk and food ration on behavior and morphology of Eurasian perch. Ecol. Evol. 2017; 7(20): 8567-77. https://doi.org/10.1002/ece3.3330.

[20] Fischer EK, Harris RM, Hofmann HA, Hoke KL. Predator exposure alters stress physiology in guppies across timescales. Horm. Behav. 2014; 65(2): 165-72.

https://doi.org/10.1016/j.yhbeh.2013.12.010.

[21] Forsythe P, Kunze WA, Bienenstock J. On communication between gut microbes and the brain. Curr. Opin. Gastroenterol. 2012; 28(6): 557-62.

https://doi.org/10.1097/MOG.0b013e3283572ffa.

[22] Candela M, Biagi E, Maccaferri S, Turroni S, Brigidi P. Intestinal microbiota is a plastic factor responding to environmental changes. Trends Microbiol. 2012; 20(8): 385-91. https://doi.org/10.1016/j.tim.2012.05.003.

[23] David LA, Maurice CF, Carmody RN, Gootenberg DB, Button JE, Wolfe BE, et al. Diet rapidly and reproducibly alters the human gut microbiome. Nature. 2014; 505(7484): 559-63. https://doi.org/10.1038/nature12820.

[24] Kikuchi Y, Hayatsu M, Hosokawa T, Nagayama A, Tago K, Fukatsu T. Symbiont-mediated insecticide resistance. PNAS. 2012; 109(22): 8618-22. https://doi.org/10.1073/pnas.1200231109. [25] Kohl KD, Weiss RB, Cox J, Dale C, Denise DM. Gut microbes of mammalian herbivores facilitate intake of plant toxins. Ecol. Lett. 2014; 17(10): 1238-46. 
[26] Belkaid Y, Hand TW. Role of the microbiota in Immunity and inflammation.

Cell. 2014; 157(1): 121-41. https://doi.org/10.1016/j.cell.2014.03.011. inoculum composition affects holobiont assembly and host growth in Daphnia.

Microbiome. 2018; 6(1): 56.

[28] Chen B, Teh BS, Sun C, Hu S, Lu X, Boland W, et al. Biodiversity and activity of the gut microbiota across the life history of the insect herbivore spodoptera littoralis. Sci Rep. 2016; 6: 29505. https://doi.org/10.1038/srep29505.

[29] Moll RM, Romoser WS, Modrakowski MC, Moncayo AC, Lerdthusnee K.

Meconial peritrophic membranes and the fate of midgut bacteria during mosquito

https://doi.org/10.1603/0022-2585-38.1.29.

[30] Chen B, Du K, Sun C, Vimalanathan A, Liang X, Li Y, et al. Gut bacterial and https://doi.org/10.1111/1758-2229.12092.

Contribution of neutral processes to the assembly of gut microbial communities in the 
zebrafish over host development. ISME J. 2016; 10(3): 655-64.

https://doi.org/10.1038/ismej.2015.142.

[33] Koenig JE, Spor A, Scalfone N, Fricker AD, Stombaugh J, Knight R, et al.

Succession of microbial consortia in the developing infant gut microbiome. PNAS.

2011; 108: 4578-85. https://doi.org/10.1073/pnas.1000081107.

[34] Palmer C, Bik EM, Digiulio DB, Relman DA, Brown PO. Development of the Human Infant Intestinal Microbiota. PloS Biol. 2007; 5(7): e177.

https://doi.org/10.1371/journal.pbio.0050177.

[35] Smith CCR, Snowberg LK, Caporaso JG, Knight R, Bolnick DI. Dietary input of microbes and host genetic variation shape among-population differences in stickleback gut microbiota. ISME J. 2015; 9(11): 2515-26.

https://doi.org/10.1038/ismej.2015.64.

[36] Yang Z, Zhang L, Zhu X, Wang J, Montagnes DJS. An evidence-based framework for predicting the impact of differing autotroph-heterotroph thermal sensitivities on consumer-prey dynamics. ISME J. 2016; 10(7): 1767-78. https://doi.org/10.1038/ismej.2015.225.

[37] Yang Z, Lowe CD, Crowther W, Fenton A, Watts PC, Montagnes DJS. Strainspecific functional and numerical responses are required to evaluate impacts on predator-prey dynamics. ISME J. 2013; 7(2): 405-16.

https://doi.org/10.1038/ismej.2012.117.

[38] Stoks R, Govaert L, Pauwels K, Jansen B, De Meester L. Resurrecting complexity: the interplay of plasticity and rapid evolution in the multiple trait 
response to strong changes in predation pressure in the water flea Daphnia magna. Ecol. Lett. 2016; 19(2): 180-90. https://doi.org/10.1111/ele.12551.

[39] Stibor H, Navarra DM. Constraints on the plasticity of Daphnia magna influenced by fish-kairomones. Funct. Ecol. 2000; 14(4): 455-9.

https://doi.org/10.2307/2656538.

[40] Sakwińska O. Plasticity of Daphnia magna life history traits in response to temperature and information about a predator. Freshw. Biol. 1998; 39(4): 681-7. https://doi.org/10.1046/j.1365-2427.1998.00320.x.

[41] Gu L, Lyu K, Dai Z, Fan M, Zhu X, Wang J, et al. Predator-specific responses of Moina macrocopa to kaironmones from different fishes. Int. Rev. Hydrobiol. 2017; 102(3-4): 83-9. https://doi.org/10.1002/iroh.201601872.

[42] Sakwińska O. Persistent maternal identity effects on life history traits in Daphnia. Oecologia. 2004; 138(3): 379-86.

[43] Dodson SI, Ryan S, Tollrian R, Lampert W. Individual swimming behavior of Daphnia: effects of food, light and container size in four clones. J. Plankton Res. 1997; 19(10): 1537-52.

[44] Okazaki Y, Fujinaga S, Tanaka A, Kohzu A, Oyagi H, Nakano SI. Ubiquity and quantitative significance of bacterioplankton lineages inhabiting the oxygenated hypolimnion of deep freshwater lakes. ISME J. 2017; 11(10): 2279-93.

https://doi.org/10.1038/ismej.2017.89.

[45] Huang J, Xu X, Li D, Sun Y, Gu L, Zhang L, et al. Decreased calcium concentration interferes with life history defense strategies of Ceriodaphnia cornuta 
in response to fish kairomone. Limnol. Oceanogr. 2021.

https://doi.org/10.1002/lno.11876.

[46] Sun Y, Lei J, Wang Y, Cheng J, Zhou Q, Wang Z, et al. High concentration of Phaeocystis globosa reduces the sensitivity of rotifer Brachionus plicatilis to cadmium: based on an exponential approach fitting the changes in some key lifehistory traits. Environ. Pollut. 2019; 246: 535-43.

\section{https://doi.org/10.1016/j.envpol.2018.12.054.}

[47] Zhu X, Wang J, Chen Q, Chen G, Huang Y, Yang Z. Costs and trade-offs of grazer-induced defenses in Scenedesmus under deficient resource. Sci. Rep. 2016;

\section{6(1): 22594. https://doi.org/10.1038/srep22594.}

[48] Huang Y, Cui G, Li B, Zhu X, Yang Z. Elevated atmospheric CO2 enhances grazer-induced morphological defense in the freshwater green alga Scenedesmus obliquus. Limnol. Oceanogr. 2018; 63(2): 1004-14. https://doi.org/10.1002/1no.10715. [49] Peter H, Sommaruga R. An evaluation of methods to study the gut bacterial community composition of freshwater zooplankton. J. Plankton Res. 2008; 30(9): 997-1006. https://doi.org/10.1093/plankt/fbn061.

[50] Grossart HP, Dziallas C, Tang KW. Bacterial diversity associated with freshwater zooplankton. Environ. Microbiol. Rep. 2009; 1(1): 50-5.

\section{https://doi.org/10.1111/j.1758-2229.2008.00003.x.}

[51] Akbar S, Huang J, Zhou Q, Gu L, Sun Y, Zhang L, et al. Elevated temperature and toxic Microcystis reduce Daphnia fitness and modulate gut microbiota. Environ. Pollut. 2021; 271: 116409. https://doi.org/10.1016/j.envpol.2020.116409. 
[52] Freese HM, Schink B. Composition and stability of the microbial community inside the digestive tract of the aquatic crustacean Daphnia magna. Microb. Ecol. 2011; 62(4): 882-94. https://doi.org/10.1007/s00248-011-9886-8.

[53] Eckert EM, Pernthaler J. Bacterial epibionts of Daphnia: a potential route for the transfer of dissolved organic carbon in freshwater food webs. ISME J. 2014; 8(9):

1808-19. https://doi.org/10.1038/ismej.2014.39.

[54] Macke E, Callens M, De Meester L, Decaestecker E. Host-genotype dependent gut microbiota drives zooplankton tolerance to toxic cyanobacteria. Nat. Commun. 2017; 8. https://doi.org/10.1038/s41467-017-01714-x.

[55] Mushegian AA, Arbore R, Walser JC, Ebert DJ. Environmental sources of bacteria and genetic variation in behavior influence host-associated microbiota. Appl. Environ. Microbiol. 2019; 85(8): e01547-18. https://doi.org/10.1128/AEM.01547-18. [56] Peerakietkhajorn S, Tsukada K, Kato Y, Matsuura T, Watanabe H. Symbiotic bacteria contribute to increasing the population size of a freshwater crustacean, Daphnia magna. Environ. Microbiol. Rep. 2015; 7(2): 364-72.

https://doi.org/10.1111/1758-2229.12260.

[57] Peerakietkhajorn S, Kato Y, Kasalický V, Matsuura T, Watanabe H.

Betaproteobacteria Limnohabitansstrains increase fecundity in the crustacean Daphnia magna: symbiotic relationship between major bacterioplankton and zooplankton in freshwater ecosystem. Environ. Microbiol. 2016; 18(8): 2366-74. https://doi.org/10.1111/1462-2920.12919.

[58] Drake HL, Horn MA. As the worm turns: the earthworm gut as a transient habitat 
for soil microbial biomes. Annu Rev Microbiol. 2007; 61: 169-89.

https://doi.org/10.1146/annurev.micro.61.080706.093139.

[59] Anza T. Differences in gut microbiota in rat models of cardiovascular disease.

University of the Witwatersrand, 2018.

[60] Hu B, Liu X, Yin S, Fan H, Feng F, Yuan J. Effects of psychological stress on hypertension in middle-aged Chinese: a cross-sectional study. PLoS One. 2015; 10(6): e0129163. https://doi.org/10.1371/journal.pone.0129163.

[61] Pickering TG. Does psychological stress contribute to the development of hypertension and coronary heart disease? EurJ. Clin. Pharmacol. 1990; 39: S1-S7. [62] Knowles SR, Nelson EA, Palombo EA. Investigating the role of perceived stress on bacterial flora activity and salivary cortisol secretion: A possible mechanism underlying susceptibility to illness. Biol. Psychol. 2008; 77(2): 132-7.

https://doi.org/10.1016/j.biopsycho.2007.09.010.

[63] Bailey MT, Dowd SE, Galley JD, Hufnagle AR, Allen RG, Lyte M. Exposure to a social stressor alters the structure of the intestinal microbiota: implications for stressor-induced immunomodulation. Brain Behav. Immun. 2011; 25(3): 397-407. https://doi.org/10.1016/j.bbi.2010.10.023.

[64] Langille MGI, Zaneveld J, Caporaso JG, Mcdonald D, Knights D, Reyes JA, et al. Predictive functional profiling of microbial communities using $16 \mathrm{~S}$ rRNA marker gene sequences. Nat. Biotechnol. 2013; 31(9): 814-21.

https://doi.org/10.1038/nbt.2676.

[65] Wilczynski W, Dynak P, Babkiewicz E, Bernatowicz P, Leniowski K, Maszczyk 
P. The combined effects of hypoxia and fish kairomones on several physiological and life history traits of Daphnia. Freshw. Biol. 2019; 64(12): 2204-20.

\section{https://doi.org/10.1111/fwb.13407.}

[66] Wendelaar Bonga SE. The stress response in fish. Physilogical Rev. 1997; (77): $592-616$.

[67] Cant JP, Mcbride BW, Croom WJ. The regulation of intestinal metabolism and its impact on whole animal energetics. Sci. J. Anim. Sci. 1996; 74(10): 2541.

https://doi.org/10.2527/1996.74102541x.

[68] Demott WR, Mckinney EN, Tessier AJ. Ontogeny of digestion in Daphnia: implications for the effectiveness of algal defenses. Ecology. 2010; 31(2): 540-8.

\section{https://doi.org/10.1890/08-2103.1.}

[69] Kashyap PC, Marcobal A, Ursell LK, Larauche M, Duboc H, Earle KA, et al. Complex interactions among diet, gastrointestinal transit, and gut microbiota in humanized mice. Gastroenterology. 2013; 144(5): 967-77.

\section{https://doi.org/10.1053/j.gastro.2013.01.047.}

[70] Stearns JC, Lynch MD, Senadheera DB, Tenenbaum HC, Goldberg MB, Cvitkovitch DG, et al. Bacterial biogeography of the human digestive tract. Sci Rep. 2011; 1: 170. https://doi.org/10.1038/srep00170.

[71] Tollrian R, Harvell CD. The ecology and evolution of inducible defenses. Princeton University Press, 1999.

[72] Lass S, Spaak P. Chemically induced anti-predator defences in plankton: a review. Hydrobiologia. 2003; 491: 221-39. 
883

884

885

886

887

888

889

890

891

892

893

894

895

896

897

898

899

900

901

902

903

904

[73] Stoks R, Govaert L, Pauwels K, Jansen B, De Meester L. Resurrecting complexity: the interplay of plasticity and rapid evolution in the multiple trait response to strong changes in predation pressure in the water flea Daphnia magna.

Ecol. Lett. 2016; 19(2): 180-90. https://doi.org/10.1111/ele.12551.

[74] Walters AW, Hughes RC, Call TB, Walker CJ, Wilcox H, Petersen SC, et al. The microbiota influences the Drosophila melanogaster life history strategy. Mol. Ecol. 2020; 29(3): 639-53. https://doi.org/10.1111/mec.15344.

[75] Motiei A, Brindefalk B, Ogonowski M, El-Shehawy R, Pastuszek P, Liewenborg $\mathrm{B}$, et al. Disparate effects of antibiotic-induced microbiome change and enhanced fitness in Daphnia magna. PLoS One. 2020; 15(1): e0214833.

https://doi.org/10.1371/journal.pone.0214833.

[76] Mushegian AA, Walser JC, Sullam KE, Ebert DJ. The microbiota of diapause:

How host-microbe associations are formed after dormancy in an aquatic crustacean.

J. Anim. Ecol. 2018; 87(2): 400-13. https://doi.org/10.1111/1365-2656.12709.

[77] Fink P, Pflitsch C, Marin K. Dietary essential amino acids affect the reproduction of the keystone herbivore Daphnia pulex. PLoS One. 2011; 6(12): e28498.

https://doi.org/10.1371/journal.pone.0028498.

[78] Taipale SJ, Brett MT, Pulkkinen K, Kainz MJ. The influence of bacteriadominated diets on Daphnia magna somatic growth, reproduction, and lipid composition. FEMS Microbiol. Ecol. 2012; 82(1): 50-62.

https://doi.org/10.1111/j.1574-6941.2012.01406.x.

[79] Wacker A, Elert EV. Polyunsaturated fatty acids: evidence for non-substitutable 
905 biochemical resources in Daphnia galeata. Ecology. 2001; 82: 2507-20.

906 https://doi.org/10.1890/0012-9658(2001)082.

907 [80] Martin-Creuzburg D, Beck B, Freese HM. Food quality of heterotrophic bacteria

908 for Daphnia magna: evidence for a limitation by sterols. FEMS Microbiol. Ecol.

909 2011; 76(3): 592-601. https://doi.org/10.1111/j.1574-6941.2011.01076.x.

910 [81] Hanne D, Eckart Z, Katleen VG, De Meester L, Klaus J. Rapid Daphnia-

911 mediated changes in microbial community structure: an experimental study. FEMS

912 Microbiol. Ecol. 2002; 42(1): 137-49. https://doi.org/10.1111/j.1574-

913 6941.2002.tb01003.x.

914 [82] Otti O, Tragust S, Feldhaar H. Unifying external and internal immune defences.

915 Trends Ecol. Evol. 2014; 29(11): 625-34. https://doi.org/10.1016/j.tree.2014.09.002.

916 [83] Macke E, Callens M, Massol F, Vanoverberghe I, De Meester L, Decaestecker E.

917 Diet and genotype of an aquatic invertebrate affect the composition of free-living

918 microbial communities. Front. microbiol. 2020; 11: 380.

919 https://doi.org/10.3389/fmicb.2020.00380.

920 


\section{Supplementary Files}

This is a list of supplementary files associated with this preprint. Click to download.

- Additionalfile1.docx

- Additionalfile2.xlsx 